\title{
A Simplified Monitor Model for EMA Prognostics
}

\author{
Pier Carlo Berri ${ }^{1}$, Matteo D.L. Dalla Vedova ${ }^{1, *}$, and Paolo Maggiore ${ }^{1}$ \\ ${ }^{1}$ Politecnico di Torino, Department of Mechanical and Aerospace Engineering (DIMEAS), 10129 \\ Torino, Italy
}

\begin{abstract}
The complexity of aircraft systems is steadily growing, allowing the machine to perform an increasing number of functions; this can result in a multitude of possible failure modes, sometimes difficult to foresee and detect. A prognostic tool to identify the early signs of faults and perform an estimation of Remaining Useful Life (RUL) can allow adaptively scheduling maintenance interventions, reducing the operating costs and increasing safety [1-4]. A first step for the RUL estimation is an accurate Fault Detection \& Identification (FDI) to infer the system health status, necessary to determine when the components will no more be able to match their requirements [5]. With a model-based approach, the FDI is a model-matching problem, intended to adjust a parametric Monitor Model (MM) to reproduce the response of the system. The MM shall feature a low computational cost to be executed iteratively on-board; at the same time, it shall be detailed enough to account for a several failure modes [6]. We propose the simplification of an Electromechanical Actuator (EMA) dynamical model [7] for model-based FDI, focusing on the BLDC motor and Power Electronics, which account for most the computational cost of the original high fidelity model.
\end{abstract}

\section{Introduction}

Electromechanical Actuators (EMAs) are a class of electric-powered servomechanisms usually meant to control the position of a mechanical component, referred to as the user. These actuators are progressively replacing the more traditional hydraulic systems in aeronautical applications to take advantage of the More Electric and All Electric design philosophies [8,9]. Ascribing most of the secondary functions to the electrical system of the aircraft, the need for a hydraulic and pneumatic system can be virtually eliminated, resulting in overall weight reduction and fuel savings. However, some failure modes typical of EMAs, such as the mechanical jamming of the transmission, are particularly hazardous if those actuation systems are employed for flight critical functions. Hence, reliable prognostic tools are required to provide an accurate estimation of the system Remaining Useful Life (RUL), in order to either plan maintenance interventions or adaptively modify the mission profile according to the residual capability of the system.

\footnotetext{
${ }^{*}$ Corresponding author: matteo.dallavedova@polito.it
} 
This work focuses on the Fault Detection and Identification (FDI) phase of the prognostic workflow, aimed to isolate accurately the early signs of wear and damage of the components. In particular, we propose a simplified dynamical model of an EMA, to be used as a near real time monitor of the physical system, enabling to detect the actuator fault by comparing the response of the hardware and the monitor model. The monitor model shall be computationally light, to allow a fast execution even on the limited hardware resources available for onboard computations, but at the same time shall be able to reproduce the effects of several failure modes with high accuracy.

In some circumstances, a simplified dynamical models is preferable over the use of standard surrogate modelling techniques (such as Proper Orthogonal Decomposition (POD), Artificial Neural Networks, etc. [10-12]) because it allows to exploit the knowledge about the physics of the system, with a greater control over the model behavior and a greater consistency in the representation of particular phenomena. Moreover, surrogate modelling techniques based on Machine Learning are inherently non-deterministic and raise issues related to safety regulations if applied in flight critical situations.

We consider a typical EMA for aircraft secondary flight controls, whose general architecture is schematically represented in Figure 1. The system is composed by a BLDC permanent magnet motor with its power inverter module, which converts the electrical power supply into mechanical power. A compound planetary gearbox increases the torque of the motor to drive a ball screw, which moves the aircraft control surface. Then, an LVDT position sensor encodes the measured user position, which is compared to the commanded position by the control electronics module to compute the required torque, closing the feedback loop.

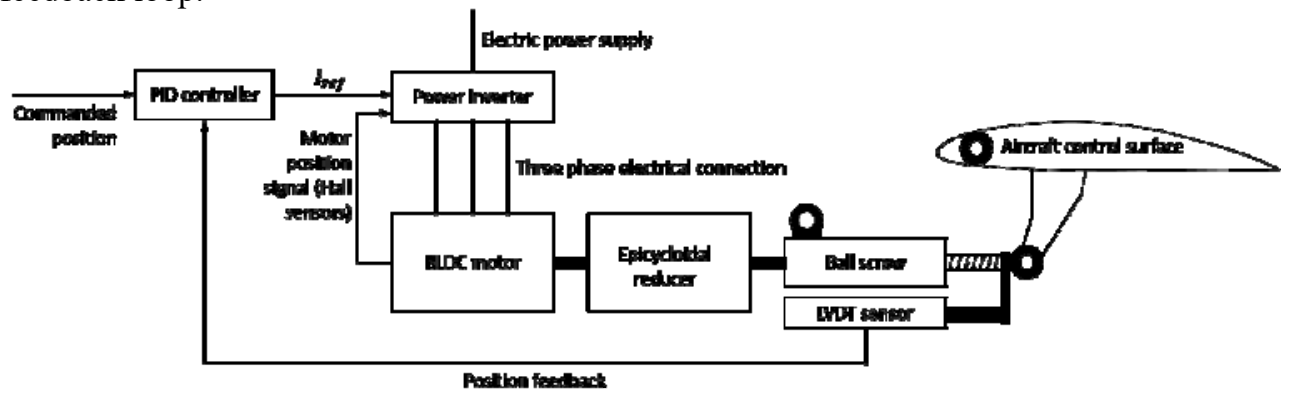

Fig. 1. Scheme of a typical EMA architecture.

The system variable monitored for the prognostic analysis is the envelope of the three phase currents; the reason is twofold:

- The three phase currents are easy to measure in a physical system, and in fact are already measured for control purposes in our application

- $\quad$ Motor current has a high sensitivity to a large variety of fault modes, so an early FDI is possible

Moreover, using the envelope of the three phases it is possible to compare the physical system and High Fidelity model (Section 2) to the single phase equivalent current of the Low Fidelity models (Section 3 and 4).

\section{Description of the High Fidelity (HF) Reference Model}

A detailed High Fidelity (HF) model of the considered EMA was initially developed by $[13,14]$. A high-level scheme of its architecture is shown in Figure 2. Each block of the HF model simulates the physical behavior of a component of the actual EMA with great accuracy, by integrating the underlying governing equations of the system. 
- $\quad$ The Control electronics block compares the measured user position (and velocity) to compute the reference current signal for the power inverter

- The Power Electronics block simulates the operation of a three-phase solid-state inverter, which applies a voltage to the active phases of the BLDC motor (according to the motor position feedback provided by the Hall sensors) trying to maintain a current equal to the commanded reference current $\left(I_{\text {ref }}\right)$.

- The BLDC Electromechanical Model contains a detailed lumped parameter simulation of the electromagnetic interactions between the rotor and stator of the electric motor. The produced torque and back-emf are computed also taking into account local inhomogeneities in the air gap width.

- The Motor-Transmission Dynamical Model computes the motor and user position by considering the system as a second order dynamical system, subject to various additional nonlinear effects: mechanical end-of-travels, static and dynamic dry friction, transmission backlash.

- The Computation of envelope current and signal filter block evaluates a filtered envelope of the three phase currents, needed to produce an output signal consistent to that of the single phase Monitor Models.

The HF model can simulate the operation of the EMA with high accuracy, however its computational cost is very high and unsuitable for nearly real time prognostics. In fact, the simulation of a 0.5 seconds test signal requires several tenths of seconds on a common laptop PC.

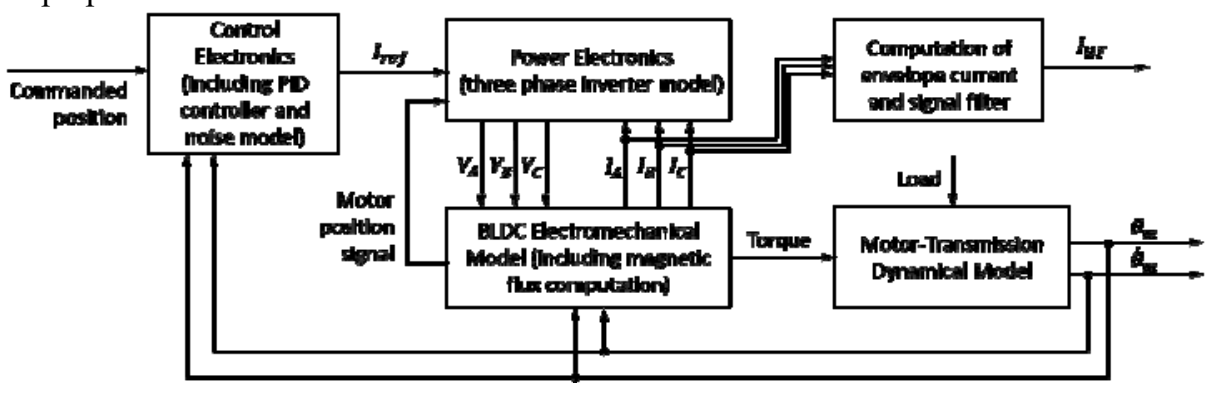

Fig. 2. Block diagram of the HF model; only the first level subsystems are shown.

\section{Description of the Old Monitor Model}

A first Low Fidelity (LF) monitor model was initially developed by [15] and modified by $[7,16]$. The model was meant to greatly reduce the computational cost of the simulation with a minimal loss of accuracy, for different position command signals and in several faulty operating conditions.

The main approximation done for this first LF model is the elimination of the static inverter model and the three-phase commutation logic. The trapezoidal BLDC motor is replaced by a first order dynamical model representing an equivalent single-phase permanent-magnet DC motor (Figure 3). Its governing equations relate the motor current $I_{m}$, the applied voltage $V$ and the produced torque $T$ :

$$
\begin{gathered}
R I_{m}+L I_{m}^{\dot{ }}=V_{m}-k_{\text {cemf }} \omega \Rightarrow \frac{\widehat{I_{m}}}{\widehat{V}-k_{\text {cemf }} \widehat{\omega}}=\frac{1 / R}{1+\frac{L}{R} s} \\
T=G_{t} I_{m}
\end{gathered}
$$

where $G_{t}=k_{\text {cemf }}=\partial \Phi / \partial \theta$ is the electromagnetic coupling constant of the motor and denotes the Laplace transform. The PWM motor control was simulated by a sign block to apply a voltage: 


$$
V=\left\{\begin{aligned}
V_{a l,} & I_{m}<I_{r e f} \\
0, & I_{m}=I_{r e f} \\
-V_{a l}, & I_{m}>I_{r e f}
\end{aligned}\right.
$$

This way, the controller tries to follow the commanded current $I_{\text {ref }}$ computed by the control electronics block, while observing the limitations imposed by the maximum value of the supply voltage $V_{a l}$. This strategy allows to reproduce the behavior of the HF model in nominal conditions. However, the introduction of electrical faults (rotor eccentricity and partial short circuit) produces ripples in the motor current with characteristic patterns that must be introduced indirectly. Two shape functions were introduced for this purpose, to correct the electrical parameters of the model according to the rotor angular position [7].

- $\quad$ Rotor eccentricity was simulated by multiplying the back-EMF coefficient $k_{\text {cemf }}$, and the torque gain $G_{t}$ by a function:

$$
f_{\zeta}\left(\theta_{m}\right)=1-0.42 \zeta\left[\cos \left(P \theta_{m}+\psi\right)+\operatorname{sawtooth}\left(6 P \theta_{m}-\pi\right) \sin \left(P \theta_{m}+\psi\right)\right]
$$

- Partial short circuit was simulated by multiplying the back-emf coefficient $k_{\text {cemf }}$, torque gain $G_{t}$ and winding resistance $R$ by a function $f_{s c}\left(\theta_{m}\right)$, expressing the fraction of functioning coils active at a time; the winding inductance $L$ is multiplied by the mean value of $f_{s c}$ :

$$
f_{s c}\left(\theta_{m}\right)= \begin{cases}\frac{N_{b}+N_{c}}{2}, & -\pi / 6 \leq \theta_{m}<\pi / 6 \\ \frac{N_{a}+N_{c}}{2}, & \pi / 6 \leq \theta_{m}<\pi / 2 \\ \frac{N_{a}+N_{b}}{2}, & \pi / 2 \leq \theta_{m}<5 \pi / 6\end{cases}
$$

where $N_{a}, N_{b}, N_{c}$ are the fraction of functioning windings of each motor phase.

The back-EMF is computed by multiplying the nominal coefficient by $f_{\bar{c}}\left(\theta_{m}\right) f_{s c}\left(\theta_{m}\right)$. The shape functions, and in particular the one relative to the rotor eccentricity, were derived with a trial and error procedure. Now we propose a more general method for their derivation that will be described in Section 4.

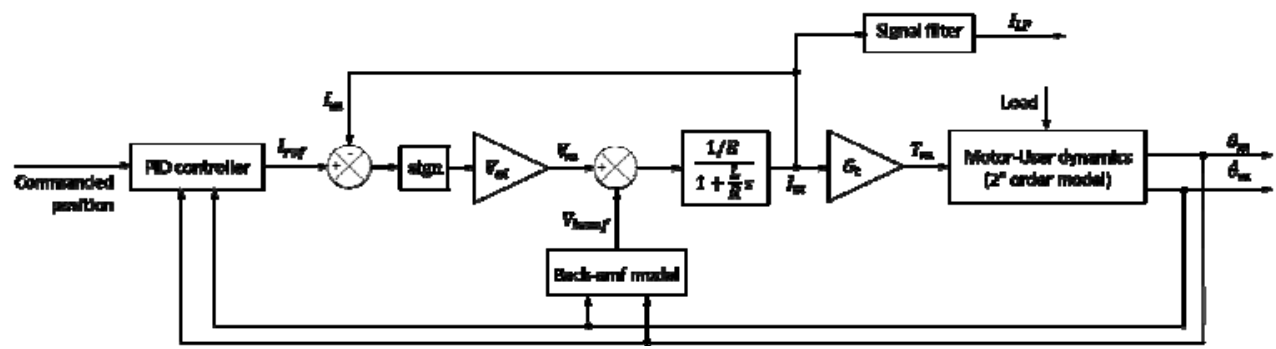

Fig. 3. Block diagram of the old LF model (with current feedback loop)

\section{Description of the Simplified Monitor Model}

The old LF model required the inductive effect of the motor windings not to produce an instantaneous current loop (see Figure 3). However, for the small and medium electric motors used for EMAs, the motor inductance is relatively low, producing very fast electrical dynamics. Then, the integration of the model requires a short time step (at most in the order of $10^{-6}$ to $10^{-5}$ seconds) to avoid numerical instabilities, resulting in a relatively high computational cost. On the other hand, the fast dynamics produced by the motor inductance has negligible effects on the overall operation of the motor. Then, it is desirable 
to neglect this phenomenon, to allow simulating a much lighter model without an excessive loss of accuracy.

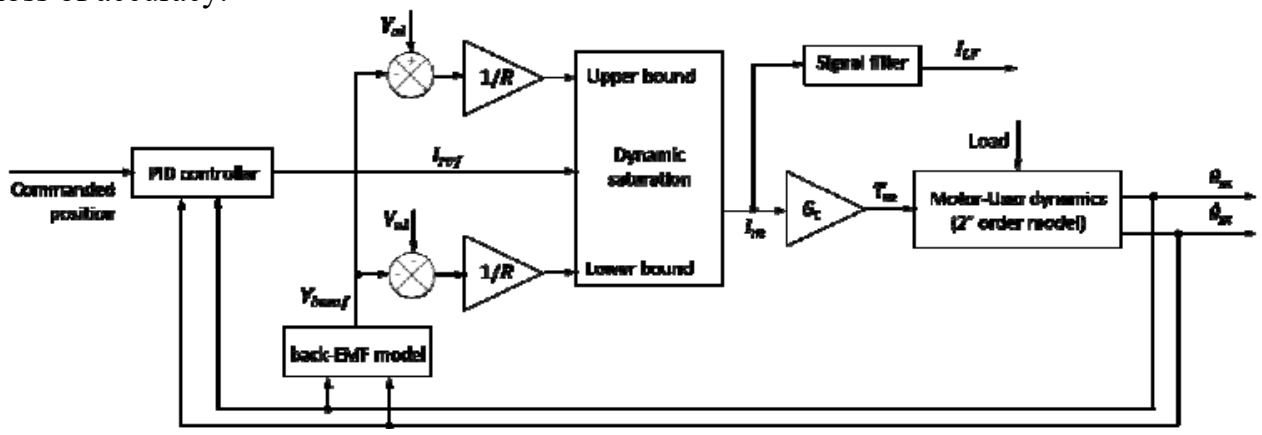

Fig. 4. Block diagram of the new LF model (with dynamic saturation)

A possible solution is to pre-solve the current loop, while keeping the same overall architecture of the old LF model. The current feedback control is meant to follow the commanded current $I_{\text {ref }}$, the time constant of this phenomenon is very small (for our particular application, in the order of $10^{-5}$ seconds). Then, we can neglect the inductive effect assuming:

$$
L=0 \Rightarrow \tau=\frac{L}{R}=0
$$

This results in $I=I_{\text {ref, }}$, when this is compatible with the maximum supply voltage available. We can notice that the effective voltage that can be applied to the motor winding is bounded between:

$$
\left\{\begin{array}{l}
V_{\max }=V_{a l}-k_{c e m f} \omega \\
V_{\min }=-V_{a l}-k_{c e m f} \omega
\end{array}\right.
$$

resulting in a maximum current of:

$$
\left\{\begin{array}{l}
I_{\max }=\frac{V_{a l}-k_{c e m f} \omega}{R} \\
I_{\text {min }}=\frac{-V_{a l}-k_{c e m f} \omega}{R}
\end{array}\right.
$$

This is equivalent to replace the whole current loop with a dynamic saturation block, as shown in Figure 4. This strategy allows adopting a longer integration time step, shortening the computational time without incurring in numerical instability problems. Another modification is the generalization of the shape functions. Starting from (1) and assuming $L=0$ we can solve for $k_{\text {cemf }}$ :

$$
k_{\text {cemf }}=\frac{V_{m}-R I_{m}}{\omega}
$$

Then, applying a ramp command to the HF model in presence of an electrical fault, we assume $\omega$ to settle to a constant value and we measure $V_{m}(t), I_{m}(t)$ and $\theta_{m}(t)$. Then, the shape function relative to the fault mode introduced in the HF model is computed as:

$$
f\left(\theta_{m}\right)=\frac{k_{c e m f}\left(\theta_{m}\right)}{k_{c e m f}^{N C}}
$$

where $k_{c e m f}^{N C}$ is the back-emf coefficient in nominal conditions; this can be expressed as a lookup table or, if feasible, approximated with a simple function (e.g. combination of sine waves and steps). 
With the dynamic saturation block for current computation, the integration time step is limited only by the cut frequency of the signal filter (Figure 4). The filter is needed in the High Fidelity model to reduce the ripple in the current signal produced by the PWM control. In the monitor models, it is necessary to introduce the same filter in order to match the signal delay and compare two consistent signals.

To further reduce the computational cost of the LF model, a first strategy consists in tuning the filter cut frequency, in order to find the maximum characteristic time that produces an acceptable loss of information. Another strategy is to translate the model from the Simulink environment to a Matlab script, and split the integration of the model itself and the filter. This way, the model is integrated with a longer time step, while a smaller one is employed only for the filter, greatly reducing the computational time (at the expense of a slight reduction in resolution and accuracy).

\section{Model validation and results}

We define a two of performance parameters related to the ability of the monitor models to replicate the response of the high fidelity model:

- Mean Squared Error (MSE):

$$
e_{m s}=\frac{1}{I_{H F r m s}^{2}} \frac{\Delta t}{t} \sum_{i}\left(I_{L F i}-I_{H F i}\right)^{2}
$$

where $\Delta t$ is the time step, $t$ is the duration of the simulation, the subscript $i$ denotes the $i$-th point of the signal expressed as a time series and $r m s$ denotes the root mean square value of a variable.

- $\quad$ Total least squares error (TLSE) [17]:

$$
e_{t l s}=\frac{1}{I_{\text {HFrms }}^{2}} \frac{\Delta t}{t} \sum_{i} \frac{\left(I_{L F i}-I_{H F i}\right)^{2}}{\left(\dot{I}_{H F i} / \dot{I}_{H F r m s}\right)^{2}+1}
$$

where the time derivative $\dot{I}_{H F}$ is evaluated numerically.

In both the errors, the term $\Delta t / t$ is the inverse of the number of integration steps. It has to be noticed that the total least squares error has been chosen because it allows to give the same importance to errors in signal and in time. Conversely, with the more traditional mean square error, a steep derivative of the signal would magnify the discrepancy in time $[17,18]$.

To assess the performances of the models, we test them on combinations of two command signals and three load profiles, listed in Table 1, in presence of 100 random fault conditions. The response of the HF model is taken as a reference signal.

Table 1. Command and load signals.

\begin{tabular}{|lcc|llll|}
\hline \multicolumn{3}{|c|}{ Commands } & \multicolumn{4}{|c|}{ Loads } \\
\hline Ramp signal & & & Step signal & & \\
\hline Slope: & 0.5 & {$[\mathrm{rad} / \mathrm{s}]$} & Amplitude: & 100 & {$[\mathrm{Nm}]$} \\
\hline Chirp signal & & & Ramp signal & & \\
\hline Amplitude: & 0.005 & {$[\mathrm{rad}]$} & Slope: & 100 & {$[\mathrm{Nm} / \mathrm{s}]$} \\
\hline Start frequency: & 0 & {$[\mathrm{~Hz}]$} & Sine wave & & \\
\hline End frequency: & 15 & {$[\mathrm{~Hz}]$} & Amplitude: & 100 & {$[\mathrm{Nm}]$} \\
\hline Duration: & 0.5 & {$[\mathrm{~s}]$} & Frequency: & 10 & {$[\mathrm{~Hz}]$} \\
\hline
\end{tabular}

Figure 5 shows the current errors for the different combinations of loads and commands, while Figure 6 synthetizes the average computational time needed for the simulation of the three models. The computational time of the Simplified Monitor Model is reduced of more than 50\% with respect to the old Monitor Model; the speed increase with respect to the High Fidelity Model is almost three orders of magnitude. 


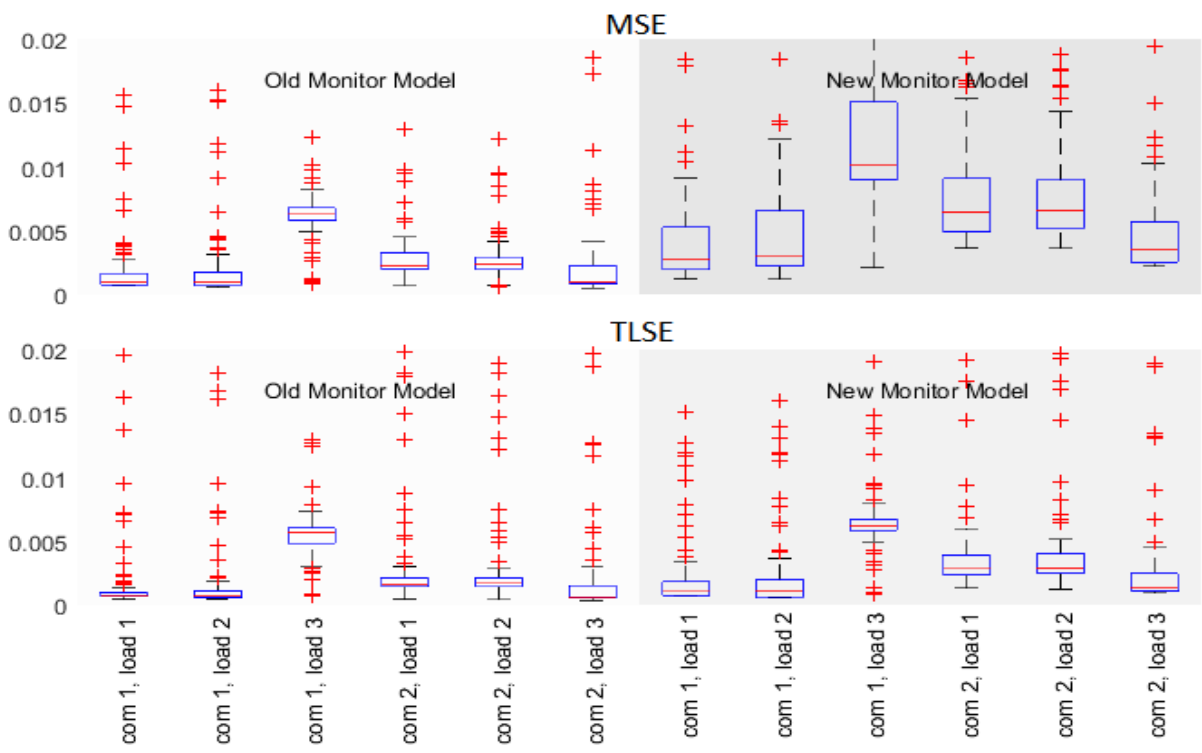

Fig. 5. Comparison of MSE (top) and TLSE (bottom) of Old and New Monitor Models

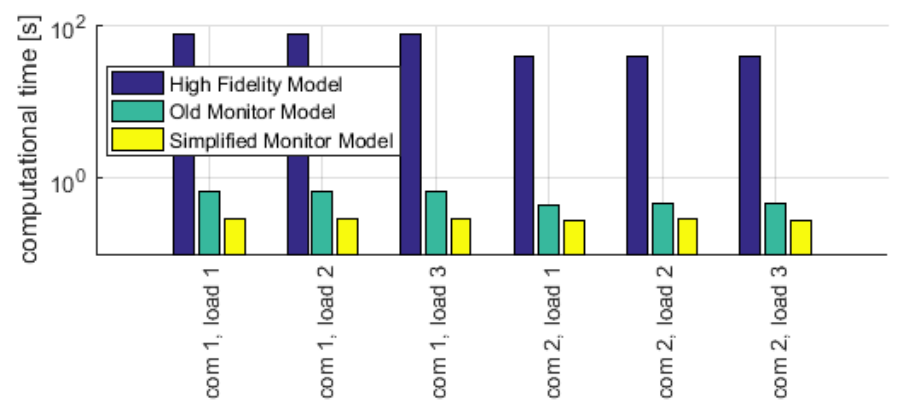

Fig. 6. Computational time needed for the three models in the considered test cases

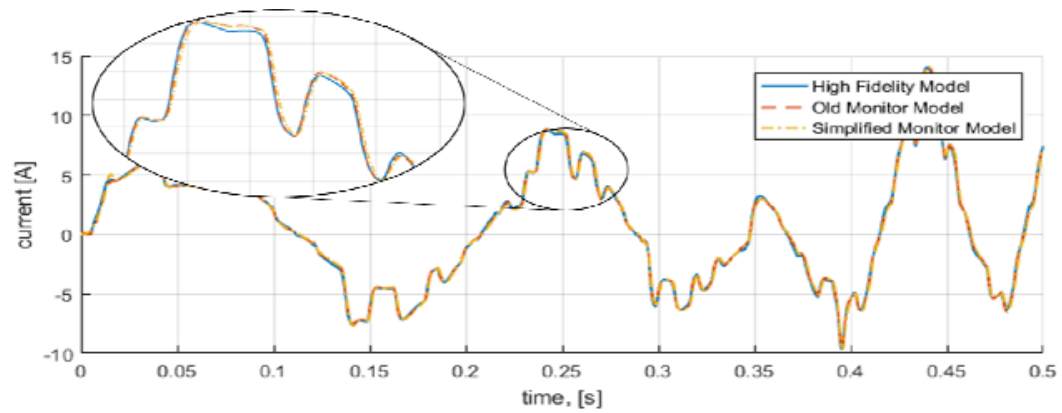

Fig. 7. Example of current signal of the three models

Comparing the accuracy of the two low fidelity models for a given combination of command signal and load profile, the Simplified Monitor Model results in a slightly higher mean squared error with a greater dispersion. However, the total least squares error is almost unchanged, suggesting a consistent behavior of the models. In fact, to match the response of the other models, the signal filter of the Simplified Model is tuned with a cut 
frequency about $20 \%$ lower. This is needed to produce a consistent overshoot but introduces a slightly increased delay, which tends to be overestimated by the MSE.

However, the errors are at most in the order of $2 \%$ and both the Low Fidelity Models can mimic the reference signal with acceptable accuracy, as shown by Figure 7. As we can see, the two LF models have similar accuracy in all the considered test cases, which cover most of the domain of applicability of the Reference Model itself. However, the new LF model is much lighter in terms of needed computational time.

\section{Conclusions}

A computationally light Low Fidelity model of an Electromechanical Actuator has been developed and tested, resulting in a much reduced computational time combined to a negligible loss of accuracy with respect to older monitoring models. The model is able to reproduce the behavior of the system in a wide range of operating conditions in terms of input command, load profile and health status. This Low Fidelity model will be employed for nearly real time online FDI, enabling the implementation of efficient and reliable prognostic methodologies.

\section{References}

1. O. Benedettini, T.S. Baines, H.W Lightfoot, R.M. Greenough, Proc. Institution of Mechanical Engineers J. of Aerospace Engineering, 2 157-170 (2009)

2. T. Sutharssan, S. Stoyanov, C. Bailey, C. Yin, J. of Engineering, 215-222 (2015)

3. Z. Williams, 2006 IEEE Aerospace Conference 9 (2006)

4. NASA-CR-192656 (1992).

5. G. Vachtsevanos F. Lewis, M. Roemer, A. Hess, B. Wu, Intelligent fault diagnosis and prognosis for engineering systems (John Wiley \& Sons, Inc., Hoboken, NJ, 2006).

6. E. Balaban, A. Saxena, K. Goebel, C.S. Byington, M. Watson, S. Bharadwaj, M Smith, Annual Conf. of the PHM Society, (27 September-1 October, San Diego, CA 2009)

7. P.C. Berri, M.D.L. Dalla Vedova, P. Maggiore, Int. J. of Mechanics and Control, 2 5966 (2016)

8. M. Howse, Power Engineer, 4 35-37 (2003)

9. R.E.J. Quigley, Proc. Eighth Annual Applied Power Electronics Conference, 906-911 (1993)

10. Z.-Q. Qu, Model Order Reduction Techniques 1-11 (2004)

11. F. Chinesta, P. Ladeveze, E. Cueto, Archives of Computational Methods in Engineering 18 395-404 (2011)

12. M.A. Bazaz, Mashuq-un-Nabi, S. Janardhanan, 2012 IEEE International Conference on Signal Processing, Computing and Control (2012)

13. M.D.L. Dalla Vedova, P. Maggiore, L. Pace, A. Desando, Int. J. of Prognostics and Health Management 1 1-13 (2015)

14. M.D.L. Dalla Vedova, A. Germanà, P. Maggiore, Proc. of the 26th European Safety and Reliability Conference ESREL 2016, 313 (2017)

15. M.D.L. Dalla Vedova, P. Maggiore, L. Pace, Int. J. of Mechanics 9 236-245 (2015)

16. M.D.L. Dalla Vedova, A. Germanà, P. Maggiore, Proc. of the Third European Conference of the PHM Society, Bilbao, 5-8 July 2016, 555-564 (2016).

17. I. Markovsky, S. Van Huffel, J. of Signal Processing $102283-2302$ (2007)

18. P.C. Berri, M.D.L. Dalla Vedova, P. Maggiore, Safety and Reliability - Theory and Applications (2017) 